\title{
The MyShake Platform: A Global Vision for Earthquake Early Warning Richard M. Allen, ${ }^{1}$ (D) Qingkai Kong, ${ }^{1}$ and Robert Martin-Short ${ }^{1}$
}

\begin{abstract}
The MyShake Platform is an operational framework to provide earthquake early warning (EEW) to people in earthquake-prone regions. It is unique among approaches to EEW as it is built on existing smartphone technology to both detect earthquakes and issue warnings. It therefore has the potential to provide EEW wherever there are smartphones, and there are now smartphones wherever there are people. The MyShake framework can also integrate other sources of alerts and deliver them to users, as well and delivering its alerts through other channels as needed. The MyShake Platform builds on experience from the first 3 years of MyShake operation. Over 300,000 people around the globe have downloaded the MyShake app and participated in this citizen science project to detect earthquakes and provide seismic waveforms for research. These operations have shown that earthquakes can be detected, located, and the magnitude estimated $\sim 5$ to $7 \mathrm{~s}$ after the origin time, and alerts can be delivered to smartphones in $\sim 1$ to $5 \mathrm{~s}$. A human-centered design process produced key insights to the needs of users that have been incorporated into MyShake2.0 which is being release for Android and iOS devices in June 2019. MyShake2.0 will also deliver EEW alerts, initially in California and hopes to expand service to other regions.
\end{abstract}

Key words: Earthquake early warning, smartphone seismic networks, earthquake detection, earthquake alerts.

\section{Introduction}

Seismology is an observational science that has always been limited by our ability to deploy sensing networks to study earthquake processes and the structure of the Earth. Earthquakes continue to have a devastating effect on even the most earthquake-prepared regions of the world, e.g. Japan following the March 11, 2011 M9.1 Tohoku-Oki earthquake. The

Electronic supplementary material The online version of this article (https://doi.org/10.1007/s00024-019-02337-7) contains supplementary material, which is available to authorized users.

1 Berkeley Seismology Lab and the Department of Earth and Planetary Science, University of California Berkeley, Berkeley, USA. E-mail: rallen@berkeley.edu
MyShake project aims to form a symbiosis between the needs of the seismology research community to collect data for all forms of research, and the needs of society to better mitigate the impacts of earthquakes. MyShake achieves this goal by turning personal/private smartphones into sensors collecting earthquake data, and delivering earthquake information to the user before, during and after an event, including earthquake early warning.

Earthquake early warning (EEW) is the rapid detection of an earthquake, decision about the region to alert, and delivery of an alert to people and automated systems in that region. The development and implementation of EEW has been accelerating with the advance of communications technologies but has been limited to the regions with seismic networks. For reviews of EEW development and implementation see Allen et al. (2009) and Allen and Melgar (2019).

The public warning systems in Mexico (Cuellar et al. 2014; Allen et al. 2017), Japan (Hoshiba 2014), South Korea (Sheen et al. 2017) and Taiwan (Wu et al. 2013, 2016; Hsu et al. 2016) show that the most common and widespread use of EEW is personal alerting for personal protective actions (Allen and Melgar 2019). For many, the response is simply to drop, cover and hold on, but this also includes improving workplace safety for workers in hazardous environments. Other common actions are the automated slowing of trains, opening and closing of pipeline valves and the readying of emergency response equipment and personnel (Strauss and Allen 2016).

Sensing technologies are also becoming much more pervasive, and the concept of the "Internet of Things" describes a world where billions of sensing devices share data in real-time around the globe. For seismologists, the use of accelerometers in a wide 
range of consumer electronics has driven the development of low-cost devices that the research community has been exploring (Allen 2012). These networks have made use of the MEMS accelerometers in laptop computers, or placed in specially installed boxes that can be easily deployed in homes and offices to detect earthquakes (Cochran et al. 2009; Chung et al. 2011; Clayton et al. 2012, 2015; Minson et al. 2015; Wu et al. 2016, 2019; Brooks et al. 2017).

The MyShake project does not purchase any sensing hardware and does not deploy or maintain any sensors. The sensing hardware is provided by smartphone owners, and the deployment process is facilitated by the Google Play and Apple iTunes stores. This approach removes significant cost while potentially provided access to $\sim 3$ billion smartphone sensors. This number will likely only grow as smartphones also become the primary means of connecting to the internet in the developing world. The price is that the sensor network is non stationary in every possible way. Users decide to join and leave the network at will by installing and uninstalling the app. The phones move around for some parts of the day, meaning that the vast majority of motions recorded by the accelerometer are not earthquakes. So, the system must effectively filter this out. MyShake must also achieve this while not impacting the normal daily use of the phone. Most notably this means that the app must use minimal power.

There are other earthquake related projects that use smartphone apps or related technologies such as social media and other forms of internet data collection. Perhaps the most familiar is the USGS "Did You Feel it?" (DYFI) which collects user felt reports via the internet (Atkinson and Wald 2007; Wald et al. 2012). The use of twitter messages sent by users experiencing earthquakes has also been explored (Earle 2010). At the European Mediterranean Seismological Centre (EMSC) multiple sources of crowdsourced information are combined to detect earthquakes. The EMSC LastQuake app provides earthquake information and collects similar experience data to DYFI. By monitoring for increased traffic to their website, use of their app and twitter, the EMSC can rapidly recognize and locate earthquakes (Bossu et al. 2018; Steed et al. 2019). The
Earthquake Network app uses the smartphone accelerometer to detect sudden movements of a phone and sends these trigger times and locations to a server which then attempts to detect and locate earthquakes based on clusters of triggers (Finazzi 2016). The Earthquake Network and MyShake apps are the only apps that use smartphone accelerometers to detect earthquake ground shaking. What is unique about MyShake is that the on-phone app attempts to distinguish earthquake ground motions from everyday motions and records $5 \mathrm{~min}$ of accelerometer data when the motion is classified as an earthquake.

In this paper we present an overview of what we refer to as the MyShake Platform. This is a technological framework to facilitate EEW in multiple regions around the world. The MyShake Platform can provide a smartphone-based sensing network to generate alerts, or can receive alerts from other sources, e.g. regional seismic networks. The Platform can distribute these alerts to smartphones or push them to other dedicated alerting systems. We first summarize the current status of the global MyShake network, and then describe the functional components of the MyShake Platform. We then review the operational lessons from the first three years of MyShake and describe how these lessons led to the development of the new MyShake system that we refer to as MyShake2.0.

\section{The MyShake Global Smartphone Seismic Network}

The MyShake smartphone app turns personal/ private smartphones into seismic sensors. Smartphone owners must first download the free app from the Google Play store (and now from the Apple iTunes store). Once installed on a phone, the app registers with the MyShake servers that operate in the cloud. The phone then becomes a sensor that is part of the MyShake global seismic network.

Continually monitoring the accelerometer or streaming continuous data requires too much power and bandwidth to be practical. Instead, an individual phone either needs to trigger or be triggered to record for short periods of time. The key technology that makes the MyShake seismic network possible is an 
artificial neural network (ANN) embedded in the smartphone app that distinguishes between earthquake-like ground motions and everyday motions (Kong et al. 2016b). In testing, the algorithm was able to correctly identify earthquake-like from other types of ground motion more than $90 \%$ of the time. To detect an earthquake, first a phone must be stationary. It then starts to monitor the accelerometer for sudden motion using a STA/LTA trigger (Allen 1978). When the phone then moves, the ANN algorithm classifies the motions as earthquake-like or not. If earthquakelike, a trigger message including the time, location and waveform characteristics is sent to the MyShake servers for use in real-time. The phone also records a total of $5 \mathrm{~min}$ of 3-component acceleration data which is later uploaded to the server for archival and later analysis. By using a 1-min ring buffer, the waveforms start $1 \mathrm{~min}$ before the trigger and continue until 4 min after.

The first public version of the MyShake smartphone application was released in February 2016. Public interest in the app was significantly greater than expected and over 335,000 people around the globe have installed the app to date (Fig. 1).
MyShake phones have detected over 900 earthquakes with magnitudes from M1.6 up to M7.8 (Kong et al. 2016a). Figure 2 shows an example of the distribution of MyShake phones triggered by a M4.4 earthquake. The 5-min waveforms can be used for a variety of purposes. These include traditional regional seismic network type of operations such as earthquake detection, location and magnitude estimation (Kong et al. 2019). The dense arrays of phone sensors can also be used to generate ShakeMaps. The recorded MyShake data captures both the response of the earth to earthquake excitation and also the response of the buildings. This means that in addition to earthquake source parameters, the characteristics of the buildings can also be determined (Kong et al. 2018). Finally, phones can be remotely triggered to record for short periods of time, which allows the phones to be used as an array. Initial evaluation of the capability suggests that the MyShake network could detect and locate earthquakes as small as M1 using this approach (Inbal et al. 2019). This is smaller than the events that traditional regional seismic networks can typically detect and could assist in the

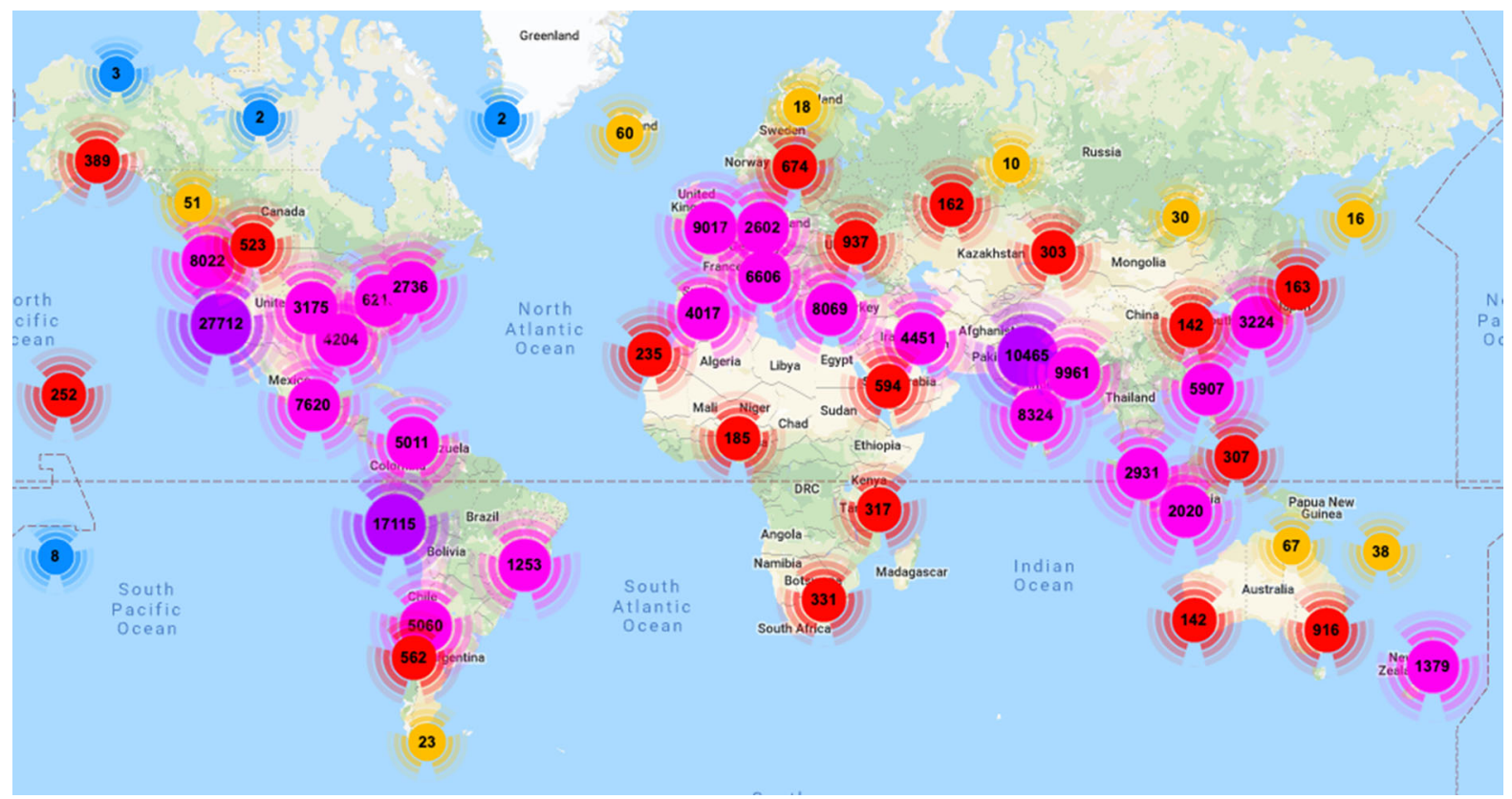

Figure 1

Map illustrating the global distribution of MyShake usage. Plotted are the locations of MyShake phones at the time they register. The locations are gathered into clusters (colored dots) and the number of phones in the cluster is shown 


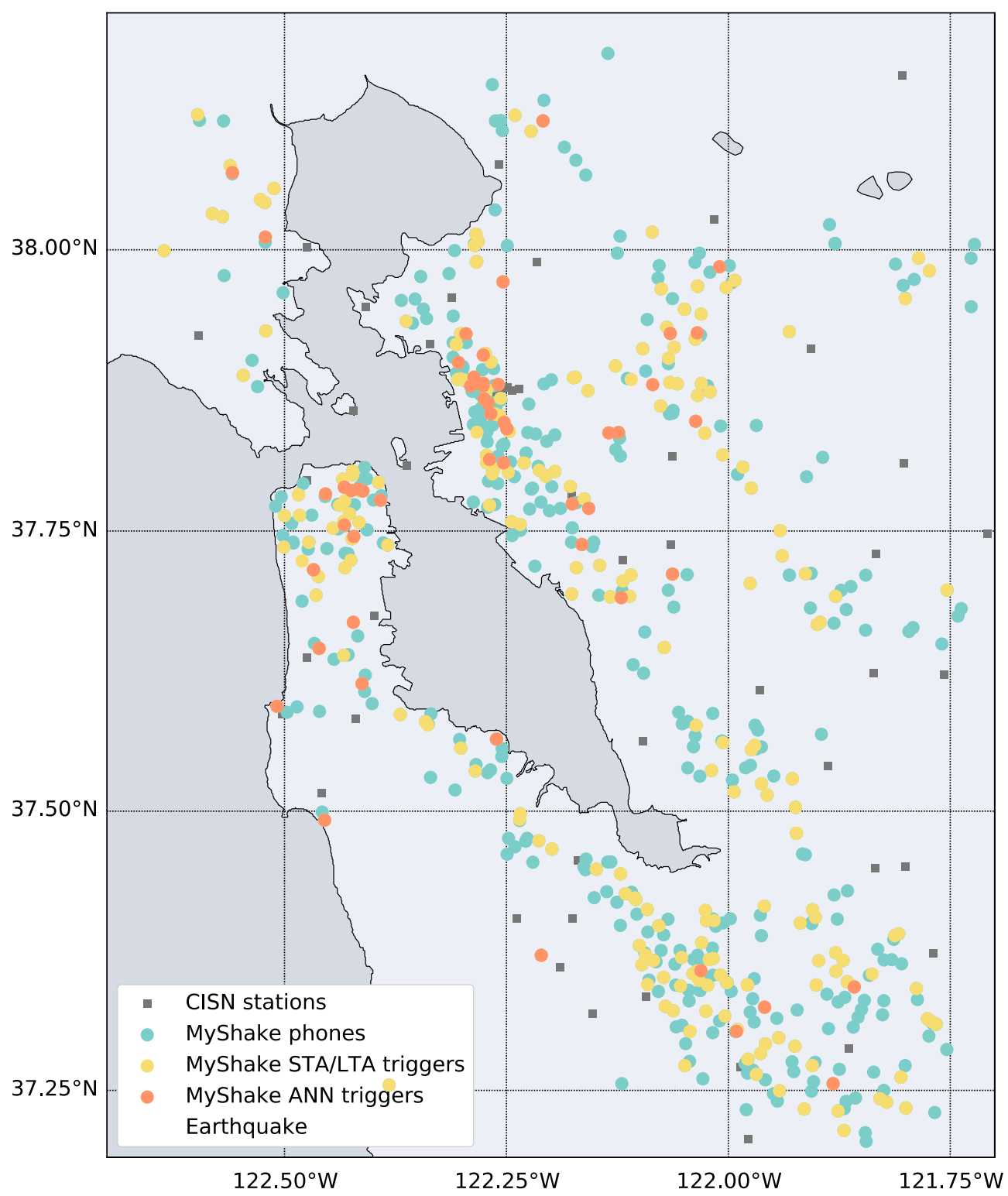

Figure 2

Map of the San Francisco Bay Area showing the locations of the 593 active MyShake phones at the time of the January 4, 2018 , M4.4 Berkeley earthquake (colored dots). At the time of the earthquake, 264 of the stationary phones triggered due to sudden motion (STA/LTA trigger-yellow dots), and the ANN algorithm recognized the shaking as earthquake-like on 52 of these phones (orange dots). The ability of the ANN algorithm to recognize earthquake-like shaking reduces rapidly for earthquakes below M5.0 which is the reason the ratio is low for this earthquake. Also shown are the 54 traditional seismic sensors of the CISN that contribute to the ShakeAlert warning system

identification of faults and hazard beneath urban environments around the world.

While the MyShake network is a global seismic network that records data for a range of purposes, earthquake early warning has always been a primary motivation and goal for the network. The reason is simply that if MyShake wants to make use of an individuals' smartphone resource, then MyShake needs to fulfill a need for that user. While many users downloaded earlier versions of the app simply to participate as citizen scientists and obtain basic earthquake information, providing a warning 
allowing a user to brace and protect themselves in the seconds before earthquake shaking is a good reason for anyone in an earthquake prone region to download the app.

\section{The MyShake Platform for Earthquake Early Warning}

Any earthquake early warning system requires a detection network, a data analysis and alerting decision module, and finally an alerting network (Fig. 3). Since the inception of EEW systems, alerts have been delivered to cellular devices, meaning that smartphones are a natural mechanism to deliver alerts. MyShake also creates the capability of detecting earthquakes and generating an alert using the sensors in smartphones. The "MyShake Platform" provides an operational framework to deliver EEW alerts to smartphones that can be generated by either phonebased detection or using traditional regional seismic networks. Here we outline the components of the MyShake platform and illustrate how MyShake can operate as an end-to-end early warning system or could interface with traditional earthquake detection system to deliver and possibly enhance alerts.

The smartphone-based earthquake detection starts with individual MyShake phones triggering on an earthquake and sending the trigger information (time, location and peak amplitude) to the MyShake server. The server then looks for space-time clusters of triggers to confirm that an earthquake is underway. This is achieved by dividing the Earth up into $100 \mathrm{~km}^{2}$ grid cells. For a cell to be "activated" multiple phones in that cell must simultaneously trigger. The criterial for a cell to activate is that there must be a minimum number of stationary phones currently monitoring, and then a defined fraction of them must individually trigger.

Once more than two cells have activated, a modified version of the "density-based spatial clustering of applications with noise" algorithm (DBSCAN, Ester et al. 1996) is used to recognize clusters of activated cells. When a cluster is found by DBSCAN, an earthquake is declared. DBSCAN can create any number of earthquakes around the globe simultaneously and associate newly triggered phones to an existing earthquake as time progresses. The earthquake is located based on the individual phone

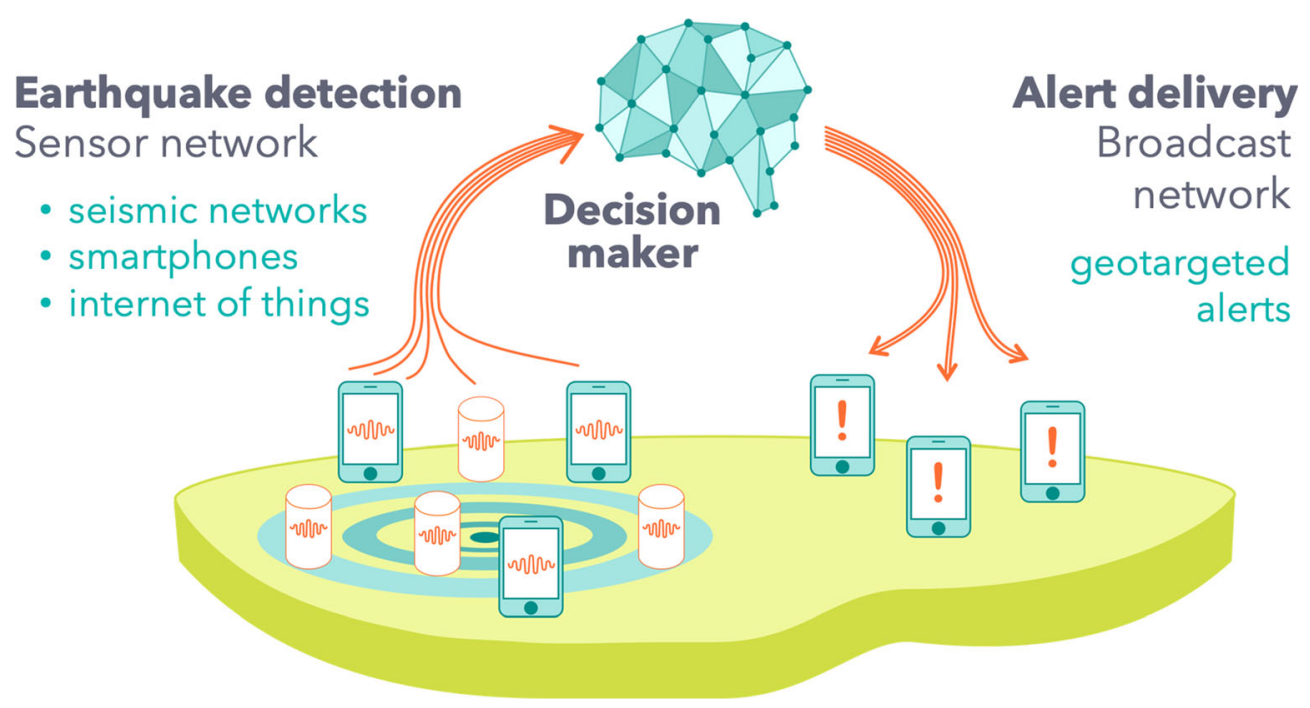

Figure 3

Schematic figure showing the components of the MyShake Platform. All earthquake early warning systems must have a sensor network to detect earthquakes (left), a decision maker that analyses the data and decides on when to alert a specific region (center), and an alert delivery network (right). MyShake can use smartphones for all of these tasks. It can also interface with other sources of alerts or deliver alerts through other non-smartphone distribution networks 
trigger times using standard techniques. The magnitude is estimated based on the peak amplitude observed by the phones at the time of the trigger. We use a random forest regressor to estimate the magnitude. This approach to event classification is described in detail in Kong, Martin-Short and Allen (in review), and is currently undergoing testing on the real-time system. What has become clear over the first few years of MyShake operation is that the MyShake network is very heterogeneous and dynamic i.e. time-dependent. As the network grows, the earthquake detection algorithm will undoubtedly have to develop with it.

The MyShake platform can receive earthquake source information from any source, not just from the MyShake phone network. For example, the MyShake platform currently receives an earthquake feed from the ShakeAlert system in the United States. ShakeAlert uses traditional seismic network stations to detect earthquakes, locate them and estimate their magnitude (Chung et al. 2019). It then generates "ShakeAlerts" for events greater than M3.5 in its operational region of California, Oregon and Washington (Kohler et al. 2017).

Once an earthquake has been detected and characterized (typically location, origin time and magnitude) by MyShake, ShakeAlert or some other system, the alert region can be determined. The criteria for issuing alerts varies from region to region and is typically chosen by regional emergency managers in consultation with the seismologists operating the warning system. In Mexico for example, any event detected by the SASMEX system with an estimated $M>6.0$ results in alerts across various cities. In Japan, alerts are issued to sub-prefectures when the expected shaking intensity exceeds 5-lower. In the US, the goal is to issue public alerts to the region expected to experience shaking intensity of MMI 3 and greater for all $M \geq 4.5$ earthquakes.

The MyShake approach to alerting uses the same $10 \mathrm{~km}$ by $10 \mathrm{~km}$ cell structure used to detect earthquakes to issue alerts. MyShake phones are dynamically registered to one of these $100 \mathrm{~km}^{2}$ cells. This provides sufficient location accuracy for geotargeted alerts, while preserving the privacy of users. When an earthquake detection is reported to the system, the alert region is defined based on the desired criteria for the region. In the case of the US, a contour is drawn around the region expected to experience MMI 3 or greater. All phones registered to a $100 \mathrm{~km}^{2}$ cell that is within or overlaps with the contour are then alerted.

The MyShake platform provides delivery of alerts to phones using standard push notification protocols provided by Google and Apple for delivery to Android and iOS apps, respectively. The alert messaging pathway starts from the MyShake servers to Google/Apple, and then on to cellular providers or through WiFi depending on how the phone is connected to the internet. The pathways to the phones are highly varied. In some cases, the MyShake servers send messages directly to individual phones. In other cases, a single message is sent to phones grouped by their coarse location and that message is directed to individual phones by a later step in the pathway. In all cases, the last step of the alert messaging pathway involves sending a message to each phone over a network connection. This raises the question of how regional cellular and $\mathrm{WiFi}$ systems will handle a message intended for a large number of phones.

There is no reason why an alert from the MyShake Platform could not also be delivered using other protocols. In South Korea and Japan, a cellular broadcast capability allows a single message to be "broadcast" simultaneously to all phones in a region. In Mexico City, sirens distributed across the city notifying people of coming shaking.

Finally, a potentially fruitful area for future development is a hybrid earthquake detection method that integrates earthquake detections from traditional seismic networks with phone-based triggers. While the detection methods using traditional seismic networks perform very well, they can still be fooled in challenging detection scenarios. These include false station triggers due to noise spikes or large teleseismic events, poorly associated triggers during intense aftershock sequences, or other unexpected forms of network noise. Likewise, the MyShake phone-based detection algorithm faces the challenges of heterogeneous phone distribution and the need for relatively high signal levels to enable detection.

Figure 2 shows the traditional seismic stations of the California Integrated Seismic Network (CISN) that are used for ShakeAlert, along with the MyShake 
phones operating at the time of the January 4, 2018, M4.4 Berkeley earthquake. ShakeAlert issued a warning when the 5 CISN stations closest to the event triggered. By the time the ShakeAlert server received these 5 triggers, the MyShake server had received 28 phone triggers. The 28 phone triggers were distributed across a much wider area than the 5 traditional sensors. Both the larger number and wider footprint of detections makes an alert that uses both networks more robust, and potentially faster.

\section{Initial Operation of MyShake}

In this section we review some of the operational details, challenges and observations from the first 3 years of operation. We cover how the app is distributed and what drives downloads. We then provide examples of earthquake detection by MyShake smartphones and illustrate the capabilities to detect and generate alerts. Finally, we provide some data on the speed with which MyShake can deliver alerts to users.

\subsection{Network Distribution}

The primary advantage of MyShake over other seismic networks is the ease with which we can "deploy" seismic sensors. A smartphone owner simply has to decide to download the app from an app store and install. The challenge is to make smartphone owners aware of the MyShake app and want to download it.

The history of MyShake app usage from initial deployment in February 2016 through June 2019 is shown in Fig. 4. Note that this history precedes the release of MyShake2.0 (discussed later), and so from a user perspective the app did not change. This initial version of MyShake that we report on was only available for Android and provided limited features for the user. These included a map of recent earthquakes, basic safety information and some information about past earthquakes of historical significance. It provided no alerts, nor any information about the earthquakes detected.

While users are downloading the MyShake app on a daily basis, interest and downloads was primarily driven by two types of events: earthquakes and media coverage. The initial release of the app was coincident with the publication of the first MyShake paper describing the earthquake recognition algorithm and was announced with a press conference. There was strong media interest and over 300 news stories were published about the app in the first few days. After 2 days the number of registered phones exceeded 50,000 and within 2 weeks it exceeded 100,000.

The number of "active" MyShake phones is the number that has provided data to the servers within the last $24 \mathrm{~h}$. It peaked 2 days after the launch at 25,000. This immediately illustrates the central challenge for MyShake: how to keep users interested so that they keep the app installed and operational on their phone for long periods of time.

Following the launch, there have been four events resulting in step-function increases in the number of users (Fig. 4). The first was May 22, 2016 when a Japanese language version of the app was released. This elicited almost no interest in Japan, but generated media interest in India where the majority of the 11,000 new downloads occurred. The second was in December 2016 and was due to a New Scientist article about the app which generated 5000 downloads, followed by media interest at the American Geoscience Union meeting that resulted in 20,000 downloads. This also took the total number of registered phones past 200,000.

The third step increase was driven by the earthquakes in Mexico in September 2017. The sequence started with a M8.1, then an M7.1 that did most damage, and finally M6.4 that generated a SASMEX warning (Allen et al. 2018). In all this resulted in about 6000 downloads. Finally, the fourth was the M8.0 beneath Peru on May 26, 2019. While it caused little damage due to its $135 \mathrm{~km}$ depth, it generated 15,000 downloads taking the total number of registered phones past the 300,000 mark.

\subsection{Earthquake Detection}

Empirical observations of the deployed MyShake network show that the on-phone earthquake detection algorithm is able to trigger and recognize earthquake ground motions from M5 earthquakes out to $\sim 250$ $\mathrm{km}$. A M4 can be detected out to $\sim 150 \mathrm{~km}$, and a 

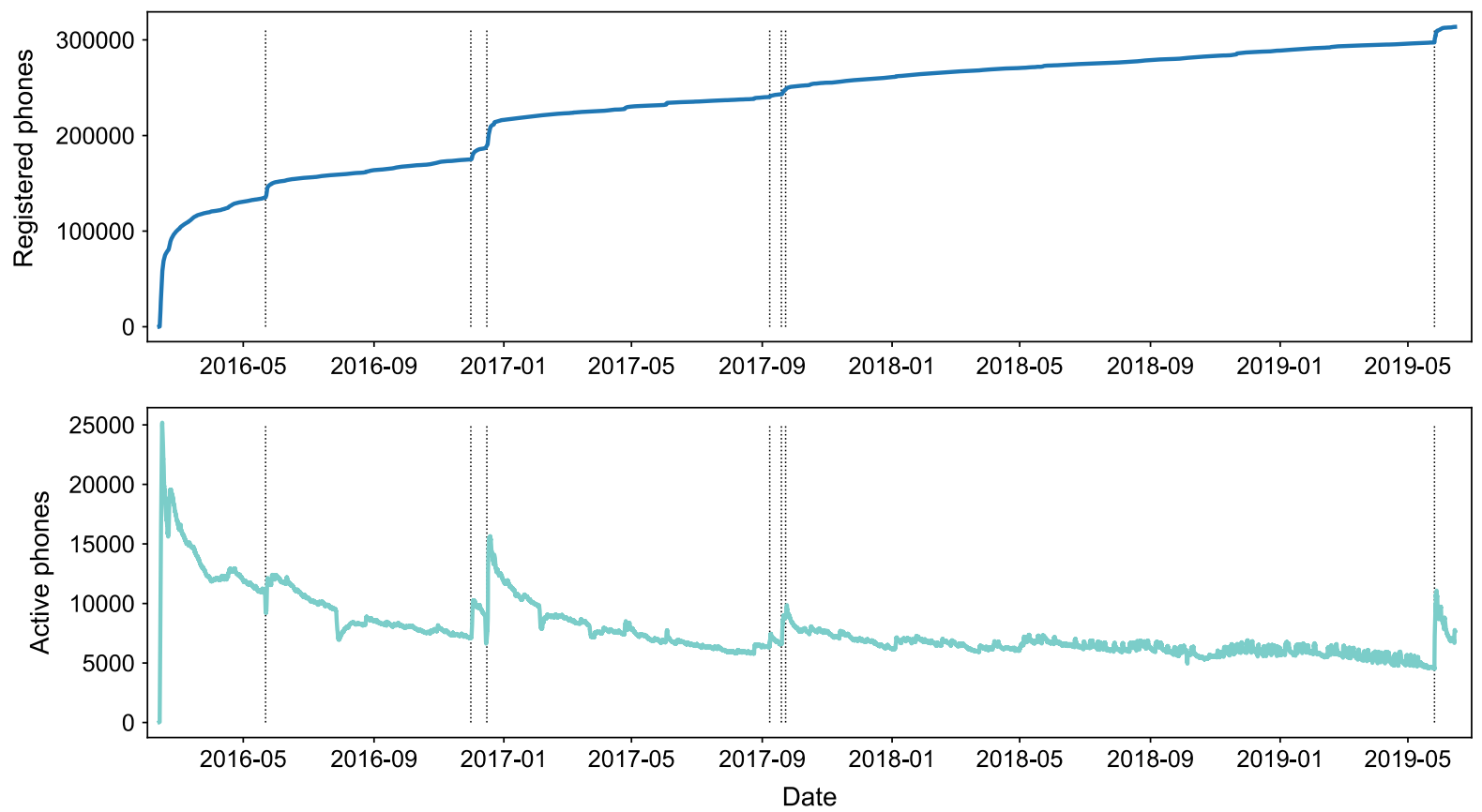

Figure 4

Plot showing the total number of registered phones (top) and the number active phones (bottom) since the launch of MyShake. The number of active phones is the number that have connected to the MyShake server within the last $24 \mathrm{~h}$. The spikes in the number of active phones (vertical dashed lines) follow significant earthquakes and/or media coverage about MyShake

M3 out to $\sim 50 \mathrm{~km}$ (Kong et al. 2019). With this detection capability, MyShake recorded ground motions from over 900 earthquakes in the first 2 years of operation.

The capability of MyShake to characterize the hypocentral parameters of the earthquake (location, magnitude and origin time) is dependent on the number and geographic distribution of MyShake phones around the event, just as it is with traditional networks. In the first 2 years of operation there were 21 earthquakes for which there were 4 or more seismic phases (P-or S-waves) detected that also had an azimuthal station/phone gap less that $180^{\circ}$. Using Hypoinverse, Kong et al. (2019) showed that the epicenter locations determined from the MyShake data had a median difference to that reported in the USGS Comcat of $2.7 \mathrm{~km}$ with a standard deviation of $2.8 \mathrm{~km}$. The depth was different by $0.1 \pm 4.9 \mathrm{~km}$ and the origin time was different by $0.2 \pm 1.2 \mathrm{~s}$. Using an $\mathrm{M}_{\mathrm{L}}$ relation based on Bakun and Joyner (1984) the median magnitude difference was $0.0 \pm 0.2$.

Figure 2 shows the detection of the January 4, 2018 M4.4 Berkeley earthquake. At the time of the event (2:40 am local time) there were 593 active MyShake phones in the Bay Area. Of these, 264 of the stationary phones triggered due to the sudden motion of the earthquake. The ANN algorithm then recognized the ground shaking as being due to an earthquake on 52 of the phones. The ANN algorithm was trained on M5.0 and larger earthquakes, so its ability to recognize shaking in smaller earthquakes is limited. While this earthquake is well recorded, it is at the lower end of the magnitude range for which we expect MyShake to perform well. The best recorded earthquake to date was the June 10, 2016, M5.2 Borrego Springs earthquake in Southern California that triggered over 200 MyShake phones and produced over 100 good seismic waveforms that show clear P-wave arrivals out to $\sim 100 \mathrm{~km}$ (Kong et al. 2016a).

\subsection{Alert Generation}

MyShake has not issued any alerts to date. However, the accumulated database of real-time earthquake detections and acceleration waveforms 
has been used to develop a new algorithm to rapidly characterize earthquakes. It is specifically tailored to the information provided by the MyShake phone detections so that an alert could be issued and limited to the geographic region expected to feel shaking. Using the MyShake observational archive, a simulation platform has been created to mimic the alert performance of a MyShake network. Thousands of simulations were then run with many instances for each earthquake using catalogs of past earthquakes around the world. The alerting algorithm and simulation platform are described in detail in Kong, Martin-Short and Allen (in review).

Here we illustrate the possible performance of MyShake-generated alerts using the September 28, 2018, M7.4 Palu earthquake on the Indonesian island of Sulawesi using the simulation platform. This earthquake and tsunami killed over 2000 people in a region prone to earthquakes. It is estimates that more than $40 \%$ of the population owns a cell phone in Indonesia. This example was generated using the simulation platform assuming that $0.1 \%$ of the population have MyShake on their phones. We use this number as this is the fraction of the population that has downloaded MyShake in the Los Angeles region. It is therefore a larger number than the current number of active phones, but also seems like a realistic goal for an operational early warning system.

Figure 5 shows snapshots from the simulation, a second-by-second movie of the simulation is included in the supplemental materials. The simulation shows that individual MyShake phones start to detect the earthquake $3.8 \mathrm{~s}$ after the origin time, which is when the P-wave first arrives at the surface. At $5.2 \mathrm{~s}$ after the origin time, when 26 triggers have been reported, the system recognizes the event, locates it, and estimated the magnitude to be M7.2. This is the point in time when the first alert could be issued across the region. It is $15.6 \mathrm{~s}$ before the $\mathrm{S}$-wave reaches the city of Palu where the population is densest and is before the S-wave has reached the surface of the Earth. Based on the estimated magnitude and location, the predicted shaking intensity in Palu is MMI 7. The observed intensity was MMI 8.

\subsection{Alert Delivery}

While MyShake has not yet delivered any alerts that are visible or apparent to the users, MyShake has sent silent alerts to MyShake phones to evaluate the delivery latency. Figure 6 shows the distribution of alert latency for a test in which a silent alert was sent to just over 100 MyShake phones in the San Francisco Bay Area. The alert latency is the time from when the alert is sent by the MyShake servers to the time that it is received by the phone. This information is reported by each phone receiving the alert. The media delay was $2.8 \mathrm{~s}$. This test was done using the current Google Firebase messaging protocol.

It is unclear how this latency will scale with the number of phones, but it should be expected to increase. The alert delivery pathway starts with the MyShake servers identifying which phones (based on location) should receive the alerts. The message is then passed to Google or Apple, who then process and pass the message to the smartphone service provider who deliver it to a specific phone. The total latency is therefore dependent on the infrastructure of multiple groups. While it would be ideal for alerts to be delivered in less than a second, we should be accepting of larger delays initially. A 2018 test of the US Wireless Emergency Alerts (WEA) system operated by the Federal Emergency Management Agency (FEMA) - commonly known as Presidential or Amber alerts-had a median delay of $13 \mathrm{~s}$ as determined by the ShakeAlert project (Douglas Given, personal communication). Despite this delay, the California Office of Emergency Management (CalOES) has determined that this is still a useful path to deliver ShakeAlerts. Thus, while we would prefer the MyShake alert delivery to be faster than $2.8 \mathrm{~s}$, it is still likely useful if it takes a little longer.

\section{MyShake2.0: Improved Features and User Interface}

MyShake is built on the participation of users. Its success as a global network and its utility for users themselves is reliant on user engagement and continued involvement. In recognition of this fact, we have taken a human-centered design approach to 

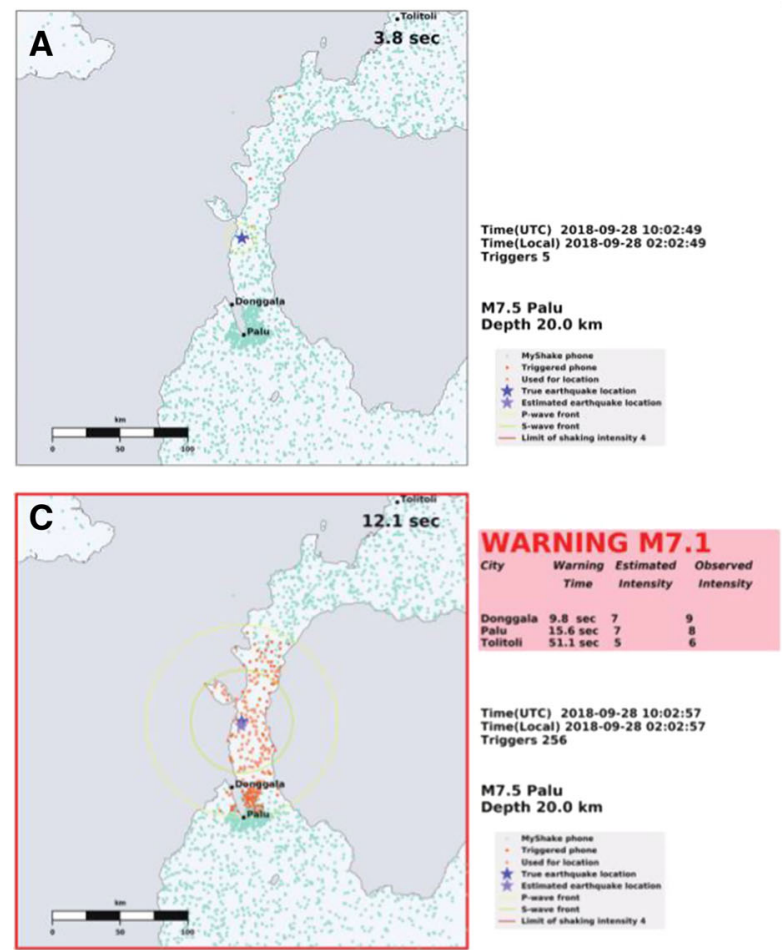
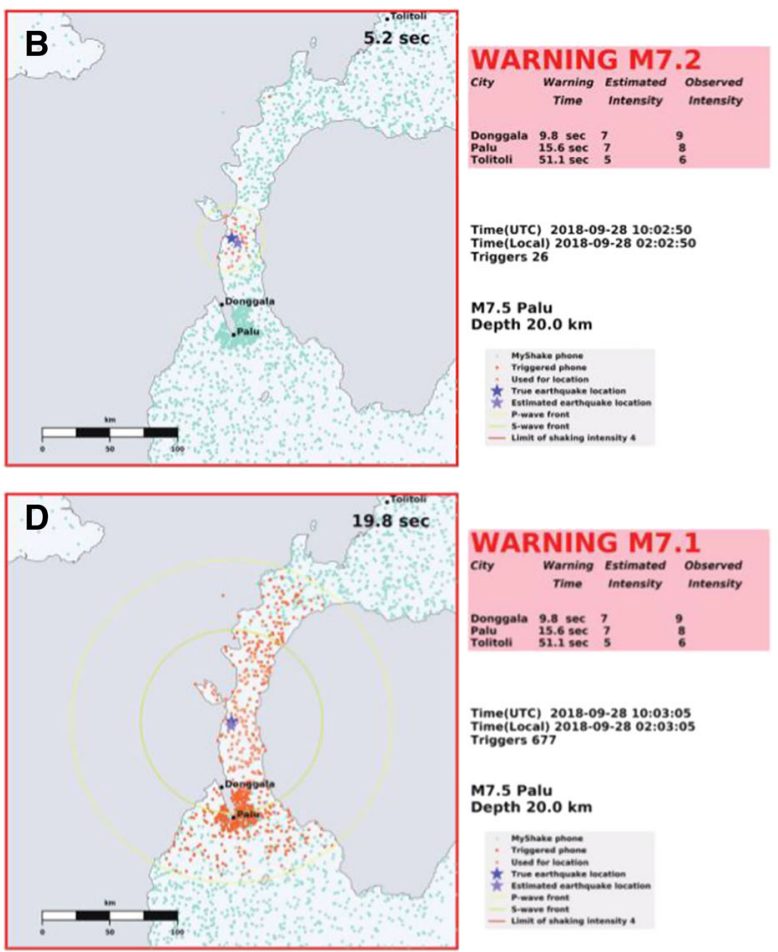

Figure 5

Snapshots from the simulation of the September 28, 2018, M7.4 Palu earthquake in Indonesia. The green dots indicate simulated MyShake phones and the blue star is the epicenter. Phone locations represent a randomly selected sample equivalent to $0.01 \%$ of the population. The population is densest in the city of Palu. a $3.8 \mathrm{~s}$ after the origin time. The P-wave reaches the surface (yellow circle) and starts to trigger some of the MyShake phones (orange dots). Note the two orange dots some distance from the earthquake. These are random phone triggers that are also modeled by the simulation platform. b $5.2 \mathrm{~s}$ after the origin time. A total of 26 phone triggers (orange dots) have reported at this time, and the network detection algorithms recognizes an earthquake is underway. It is located (light blue star) and the magnitude estimated to be M7.2. This is the first point in time when an alert could be issued of coming ground shaking across the region. The estimated shaking intensity in Palu is MMI 7 (actual intensity was MMI 8) and it is $15.6 \mathrm{~s}$ until the S-wave will arrive in Palu. The S-wave has not yet reached the surface of the Earth. c $12.1 \mathrm{~s}$ after the origin time. This is when the P-wave reaches the center of Palu. The inner yellow circle is the S-wave. Many additional phones have triggered, and the location and the magnitude updated. d $19.8 \mathrm{~s}$ after the origin time. Approximately when the S-wave reaches the center of Palu. The full simulation results are available in the movie file included as an electronic supplement (Allen-MyShake-

Palu_0.001.mp4). Additional examples of actual and simulated performance are included in Kong, Martin-Short and Allen (in review)

proposing and developing the features to be included in the next generation of MyShake. We have also redesigned the user interface. We engaged with MyShake users to understand user needs, behaviors, and pain points in order to identify what features could be added to the app to address these needs. A description of the entire design process can be found in Rochford et al. (2018). Here we highlight some of the key learnings and how they have been mapped into the new MyShake2.0.

We identified four key insights that broadly represent how the public thinks and reacts to earthquakes based on interviews (Rochford et al. 2018):
1. Participants expressed feelings of fear toward earthquakes, as well as feeling helpless to prepare themselves for a large event.

2. Participants reported avoiding thoughts of earthquakes, only engaging with earthquake and preparedness information when they either experienced one or saw one in the news.

3. Respondents wanted information about how an earthquake affected the areas where their loved ones and homes were.

4. Respondents relied on several different resources for news after an earthquake, including social 


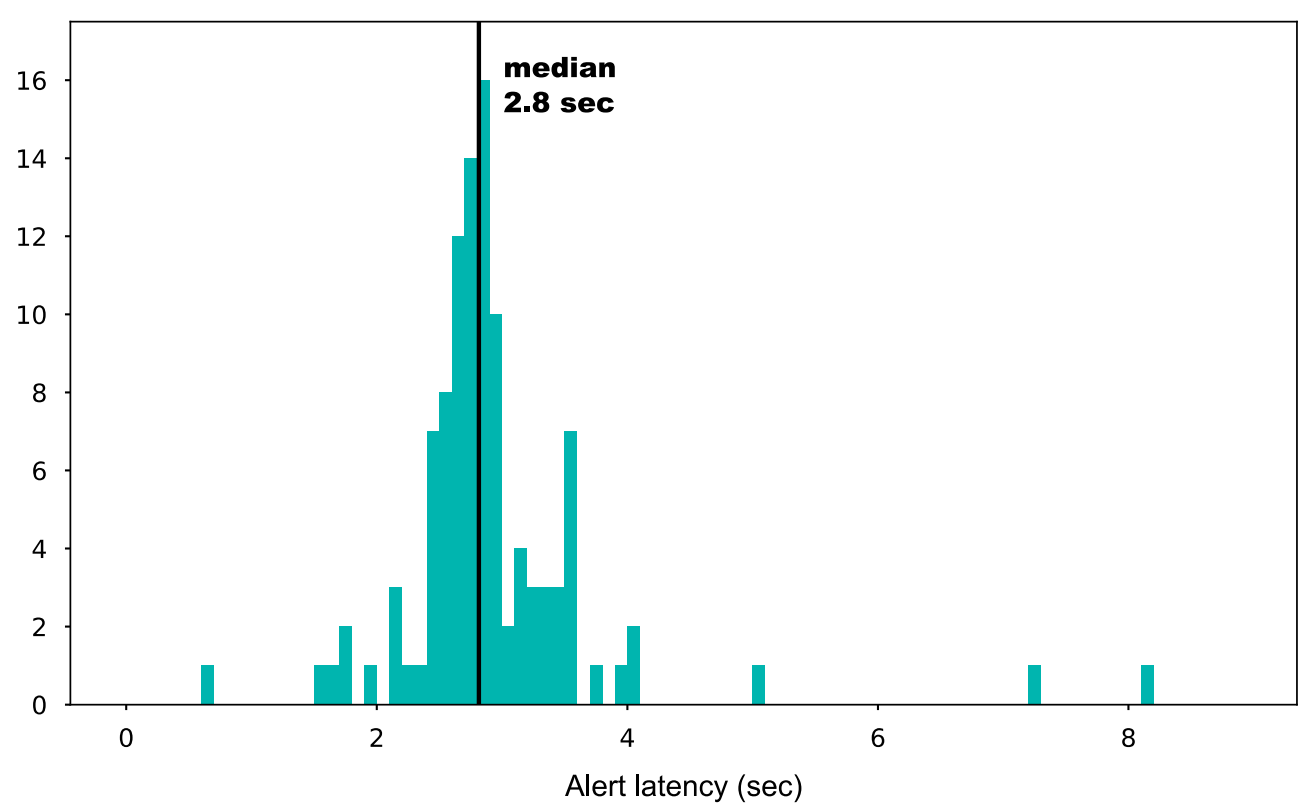

Figure 6

Observed alert latency. This is the time from when the MyShake servers sent the alert until it was received by the phones

media, local news, search engines, and the United

States Geological Survey website.

These interviews also lead to the development of a flow of actions that people took, or thought they would take, when an earthquake occurs (Fig. 7). This starts with recognition that an earthquake is underway, moves into a confirmation phase using information from various sources. Then an assessment phase were people are seeking information about what just happened and the impact. Finally, a secondary information seeking phase when people are looking for official advisory information about what to do next.

Based on the analysis of people's needs in an earthquake, and also on their reaction to participating in the MyShake project as citizen scientists, we

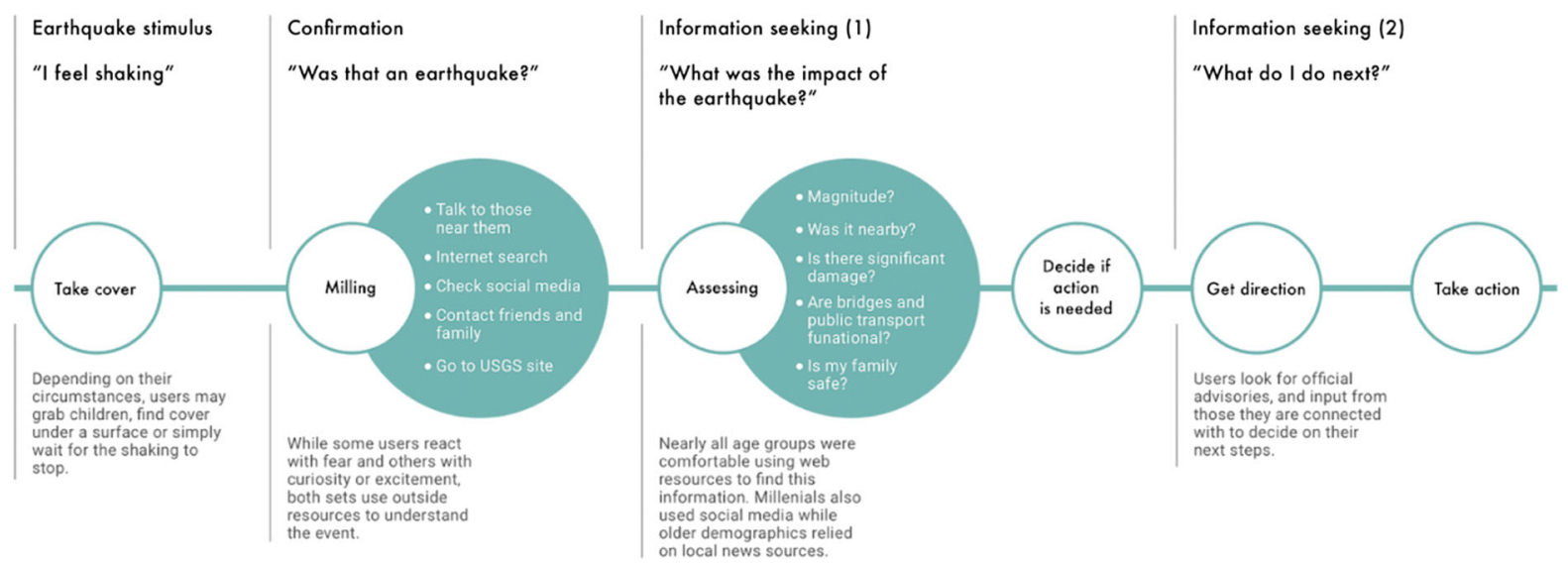

Figure 7

Flow of actions taken by people in response to an earthquake derived from interviews with MyShake users and experts (Rochford et al. 2018) 
identified features that were of greatest value to users and were also technically feasible:

1. Delivering earthquake early warning when possible so users can take protective actions in the seconds before shaking.

2. Providing highly local information about building and road damage so users can assess what actions, if any, to take in the minutes after an earthquake.

3. Proving resources that empower users to learn about earthquakes and preparedness, without inciting fear and despair.

4. Providing information about a user's contribution to citizen science through metrics and acknowledgement of their participation.

All of these features have been added to the new version of MyShake while also completely redesigning the user interface to make it both attractive and compliant with best practices in usability. We refer to this new version of the apps (Android and iOS) and system as "MyShake2.0".

MyShake2.0 will issue and receive earthquake early warning alerts. These are geotargeted alerts sent to specific phones that are located within a defined warning area. The app provides a simple interface for users to report their experience and observations when they are in an earthquake. These "experience reports" include the shaking intensity, observed building, road and bridge damage. This information is aggregated with reports from all users and is immediately displayed in the app so users can see where damage has occurred around themselves and in the locations of loved ones. There is also a "MyShake Data" page that shows the contributions made by a user to the network and also basic statistics about the network as a whole. The "My Earthquake Log" page lists a user's contributions, alerts and notifications. Finally, a "Safety" page provides straightforward information on how to prepare, survive, and recover from an earthquake with links to additional resources. The Android and iPhone apps providing all these features will be available in the Google Plan and Apple iTunes store when this article is published.

There are two additional features added to MyShake2.0 that were not an outcome of the user engagement process described above. Both are added to allow for seismological education activities in the classroom and have been developed in collaboration with the IRIS Education and Outreach program. The first is a "Sensor" page that provides a simple graphical readout of the 3-component accelerometer. It also allows this output to be recorded to a csv file that can be shared from the phone to allow analysis. The second is a simplified version of IRIS's StationMonitor [https://www.iris.edu/hq/inclass/ software-web-app/station_monitor]. This allows a user to browse earthquake waveforms from traditional/permanent seismic stations.

\section{Discussion and Future Directions}

The first 3 years of MyShake operations have demonstrated that personal smartphones can be harnessed to produce seismic waveform data that has value both for research and hazard reduction. While the response of the citizen science public to the first version of the app has been stronger than expected, it is clear that the MyShake app must be installed on many more phones in many more regions if it is to generate the necessary data to provide early warning. For this to be possible MyShake2.0 must provide greater value to users than the first version of the app.

At the time of writing, MyShake2.0 is in the final stages of testing with an expected release in June 2019. As described above, it provides a much greater range of features that have been designed specifically to address the needs of users. These will include delivery of earthquake early warning for the first time.

Initially, the app will deliver ShakeAlerts generated by traditional seismic networks to users. ShakeAlert is the US earthquake early warning system now operating in California, Oregon and Washington (Kohler et al. 2017; Chung et al. 2019). Operated by the US Geological Survey in collaboration with the University of California Berkeley, Caltech, the Universities of Washington and Oregon, and the state emergency management agencies, the ShakeAlert project determined that ShakeAlerts were ready for general use in October 2018. However, there has not been a technology solution available to deliver alerts to the broad public across all three states. The City of Los Angeles developed the 
ShakeAlertLA app that delivers alerts to the public, but only for earthquakes in the County of Los Angeles.

MyShake2.0 will initially deliver ShakeAlerts to an expanding number of test users. But, sponsored by the California Office of Emergency Services, it will deliver ShakeAlerts to the public before the end of 2019. Through this partnership with ShakeAlert, MyShake can simultaneously start delivery of alerts across the region filling ShakeAlert's technology gap, while also expanding the number of users and testing the capabilities of MyShake to generate alerts. There is no plan to issue MyShake-phone-generated alerts in the US, however we will explore how MyShakephone triggers could be used to enhance ShakeAlert for faster and more reliable alerts.

The next step for MyShake will be to start delivering phone-generated alerts in a region or regions where the density of MyShake phones is sufficient to provide reliable alerts. This will require partnership with the appropriate public safety and emergency management agencies in the region in order to ensure appropriate messaging and educational strategies to inform users.

While there remain substantial technological challenges to global delivery of earthquake early warning, our hope is that MyShake can drive the development of the necessary technologies. As MyShake capabilities improve and become more widely known, components of the MyShake Platform could be integrated with other technologies. Other popular apps could embed the MyShake detection capability within their app thus providing many more sensors without users needing to know specifically about the MyShake app and installing it. Likewise, the alerting component could be integrated into existing popular apps. An extension of this approach would be for the MyShake capability to be directly integrated into smartphone operating systems. As such, the ultimate success of the MyShake effort may be that the MyShake app is no longer needed.

\section{Acknowledgements}

The MyShake team at UC Berkeley who all contribute to this work includes Stephen Allen, Asaf
Inbal, Akie Mejia, Sarina Patel, Kaylin Rochford, Jennifer Strauss, Jennifer Taggart, Stephen Thompson, and Stephane Zuzlewski in addition to the authors. We thank John Taber and Mladen Dordevik at the IRIS Consortium for guidance on the educational components of MyShake2.0 and the creation of the StationMonitor. This work was funded by the Gordon and Betty Moore Foundation through Grant GBMF 5230 to UC Berkeley.

Open Access This article is distributed under the terms of the Creative Commons Attribution 4.0 International License (http:// creativecommons.org/licenses/by/4.0/), which permits unrestricted use, distribution, and reproduction in any medium, provided you give appropriate credit to the original author(s) and the source, provide a link to the Creative Commons license, and indicate if changes were made.

Publisher's Note Springer Nature remains neutral with regard to jurisdictional claims in published maps and institutional affiliations.

\section{REFERENCES}

Allen, R. (1978). Automatic earthquake recognition and timing from single traces. BSSA, 68, 1521-1532.

Allen, R. M. (2012). Transforming earthquake detection? Science (80-), 335, 297-298. https://doi.org/10.1126/science.1214650.

Allen, R. M., Cochran, E. S., Huggins, T. J., et al. (2018). Lessons from Mexico's earthquake early warning system. In: Eos (Washington, DC). https://eos.org/features/lessons-frommexicos-earthquake-early-warning-system. Accessed 18 Jan 2019

Allen, R. M., Cochran, E. S., Huggins, T., et al. (2017). Quake warnings, seismic culture. Science (80-), 358, 1111. https://doi. org/10.1126/science.aar4640.

Allen, R. M., Gasparini, P., Kamigaichi, O., et al. (2009). The status of earthquake early warning around the world: An introductory overview. Seismological Research Letters, 80, 682-693. https://doi.org/10.1785/gssrl.80.5.682.

Allen, R. M., \& Melgar, D. (2019). Earthquake early warning: Advances, scientific challenges, and societal needs. Annual Review of Earth and Planetary Sciences, 47, 361-388. https:// doi.org/10.1146/annurev-earth-053018-060457.

Atkinson, G. M., \& Wald, D. J. (2007). "Did you feel it?" intensity data: A surprisingly good measure of earthquake ground motion. Seismological Research Letters, 78, 362-368. https://doi.org/10. 1785/gssrl.78.3.362.

Bakun, W. H., \& Joyner, W. B. (1984). The ML scale in central California. Bulletin of the Seismological Society of America, 74, 1827-1843. 
Bossu, R., Roussel, F., Fallou, L., et al. (2018). LastQuake: From rapid information to global seismic risk reduction. IInternational Journal of Disaster Risk Reductiont, 28, 32-42. https://doi.org/ 10.1016/J.IJDRR.2018.02.024.

Brooks, B., Minson, S. E., Böse, M., et al. (2017). Towards internet of things earthquake early warning-a pilot network in Chile. Seismological Research Letters, 88, 537. https://doi.org/10.1785/ 0220170035.

Chung, A. I., Henson, I., \& Allen, R. M. (2019). Optimizing earthquake early warning performance: Elarm S-3. Seismological Research Letters, 90, 727-743. https://doi.org/10.1785/ 0220180192.

Chung, A. I., Neighbors, C., Belmonte, A., et al. (2011). The Quake-catcher network rapid aftershock mobilization program following the 2010 M 8.8 Maule, Chile earthquake. Seismological Research Letters, 82, 526-532. https://doi.org/10.1785/gssrl. 82.4.526.

Clayton, R. W., Heaton, T., Changy, M., et al. (2012). Community seismic network. Annals of Geophysics, 14, 54.

Clayton, R. W., Heaton, T., Kohler, M., et al. (2015). Community seismic network: A dense array to sense earthquake strong motion. Seismological Research Letters, 86, 1-10. https://doi. org/10.1785/0220150094.

Cochran, E. S., Lawrence, J. F., Christensen, C., \& Jakka, R. S. (2009). The Quake-Catcher network: Citizen science expanding seismic horizons. Seismological Research Letters, 80, 26-30. https://doi.org/10.1785/gssrl.80.1.26.

Cuellar, A., Espinosa-Aranda, J. M., Suarez, R., et al. (2014). The Mexican Seismic Alert System (SASMEX): Its alert signals, broadcast results and performance during the M 7.4 Punta Maldonado Earthquake of March 20th, 2012. In: Wenzel, F., \& Zschau, J. (eds) Early warning for geological disasters, pp. $71-87$

Earle, P. S. (2010). Earthquake Twitter. Nature Geoscience, 3, 221-222.

Ester, M., Kriegel, H. P., Sander, J., \& Xu, X. (1996) A densitybased algorithm for discovering clusters in large spatial databases with noise. In: Proceedings of the second international conference on knowledge discovery and data mining, pp. 226-231

Finazzi, F. (2016). The earthquake network project: Toward a crowdsourced smartphone-based earthquake early warning system. Bulletin of the Seismological Society of America. https://doi. org/10.1785/0120150354.

Hoshiba, M. (2014). Review of the nationwide earthquake early warning in Japan during its first five years. In M. Wyss (Ed.), Earthquake hazard, risk, and disasters (pp. 505-528). Oxford: Elsevier.

Hsu, T.-Y., Wang, H.-H., Lin, P.-Y., et al. (2016). Performance of the NCREE's on-site warning system during the 5 February $2016 M_{w} 6.53$ Meinong earthquake. Geophysical Research Letters, 43, 8954-8959. https://doi.org/10.1002/2016GL069372.

Inbal, A., Kong, Q., Savran, W., \& Allen, R. M. (2019). On the feasibility of using the dense MyShake smartphone array for earthquake location. Seismological Research Letters, 90, 1209-1218. https://doi.org/10.1785/0220180349.
Kohler, M. D., Cochran, E. S., Given, D., et al. (2017). Earthquake early warning ShakeAlert System: West coast wide production prototype. Seismological Research Letters. https://doi.org/10. 1785/0220170140.

Kong, Q., Allen, R. M., Kohler, M. D., et al. (2018). Structural health monitoring of buildings using smartphone sensors. Seismological Research Letters, 89, 594-602. https://doi.org/10. 1785/0220170111.

Kong, Q., Allen, R. M., \& Schreier, L. (2016a). MyShake: Initial observations from a global smartphone seismic network. Geophysical Research Letters. https://doi.org/10.1002/2016GL070955.

Kong, Q., Allen, R. M., Schreier, L., \& Kwon, Y.-W. (2016b). MyShake: A smartphone seismic network for earthquake early warning and beyond. Science Advances, 2, e1501055. https://doi. org/10.1126/sciadv.1501055.

Kong, Q., Martin-Short, R., \& Allen, R. M. Towards global earthquake early warning with the MyShake smartphone seismic network (in review)

Kong, Q., Patel, S., Inbal, A., \& Allen, R. M. (2019) Assessing the sensitivity and accuracy of the MyShake smartphone seismic network to detect and characterize earthquakes. https://arxiv.org/ abs/1904.09755

Minson, S. E., Brooks, B. A., Glennie, C. L., et al. (2015). Crowdsourced earthquake early warning. Science Advances. https://doi.org/10.1126/sciadv.1500036.

Rochford, K., Strauss, J. A., Kong, Q., \& Allen, R. M. (2018). MyShake: Using human-centered design methods to promote engagement in a smartphone-based global seismic network. Frontiers in Earth Science, 6, 1-14. https://doi.org/10.3389/feart. 2018.00237.

Sheen, D., Park, J., Chi, H., et al. (2017). The first stage of an earthquake early warning system in South Korea. Seismological Research Letters, 88, 1491-1498. https://doi.org/10.1785/ 0220170062.

Steed, R. J., Fuenzalida, A., Bossu, R., et al. (2019). Crowdsourcing triggers rapid, reliable earthquake locations.

Strauss, J. A., \& Allen, R. M. (2016). Benefits and costs of earthquake early warning. Seismological Research Letters, 87, 765-772. https://doi.org/10.1785/0220150149.

Wald, D., Wald, D. J., Quitoriano, V., et al. (2012). USGS “Did You Feel It?" Internet-based macroseismic intensity maps. Annales Geophysicae. https://doi.org/10.4401/ag-5354.

Wu, Y.-M., Hsiao, N.-C., Chin, T.-.L, et al. (2013). Earthquake early warning system in Taiwan. In: Encyclopedia of earthquake engineering.

Wu, Y., Liang, W., Mittal, H., et al. (2016). Performance of a lowcost earthquake early warning system (P-Alert) during the 2016 M L 6.4 Meinong (Taiwan) Earthquake. Seismological Research Letters, 87, 1050-1059. https://doi.org/10.1785/ 0220160058 .

Wu, Y., Mittal, H., Huang, T., et al. (2019). Performance of a lowcost earthquake early warning system (P-Alert) and Shake Map Production during the $2018 \mathrm{Mw} 6.4$ Hualien, Taiwan, Earthquake. Seismological Research Letters, 90, 19-29. https://doi. org/10.1785/0220180170. 